15

\title{
Тепловой режим самонакаливаемого полого катода в сильноточном импульсно-периодическом разряде низкого давления
}

\author{
(C) Н.В. Гаврилов, Д.Р. Емлин \\ Институт электрофизики УрО РАН, 620016 Екатеринбург, Россия \\ e-mail: gavrilov@iep.uran.ru
}

(Поступило в Редакцию 3 марта 2017 г.)

Изучены тепловой режим полого самонакаливаемого катода при совместном горении слаботочного (1-5 A) разряда постоянного тока и сильноточного (до $100 \mathrm{~A}$ ) импульсно-периодического разряда и влияние импульсных параметров на вольтамперную характеристику сильноточного разряда. Показано, что после приложения импульса напряжения $(200-500 \mathrm{~V})$ за время $\sim 100 \mu \mathrm{s}$ ток разряда достигает максимума и стабилизируется. Напряжение горения разряда в квазистационарной стадии разряда многократно превышает напряжение горения непрерывного разряда с тем же током и зависит от средней величины тока в разрядном промежутке. Дано объяснение характера вольтамперных характеристик импульсного разряда, основанное на динамике нагрева и остывания поверхностного слоя катода и изменении интегральной температуры катода.

DOI: 10.21883/JTF.2017.11.45141.2230

\section{Введение}

Импульсные свойства разряда с самонакаливаемым полым катодом определяются временем достижения установившегося состояния в результате самосогласованного протекания процессов ионизации газа в объеме катодной полости, нагрева катода ионной бомбардировкой и электронной эмиссии катода. Малый объем катодной полости и повышенное давление газа обеспечивают высокую частоту ионизации газа осциллирующими электронами, поэтому скорость развития такого разряда определяется тепловыми процессами на катоде. Оценки теплового режима катода из нитрида титана показали, что температура поверхности катода может быть увеличена на $200-600^{\circ} \mathrm{C}$ за время $\sim 10^{-4}$ s при плотности мощности на поверхности катода $10^{3}-5 \cdot 10^{4} \mathrm{~W} / \mathrm{cm}^{2}[1]$. Достаточно быстрый нагрев обусловлен выделением тепла в приповерхностном слое толщиной в несколько десятков микрометров. В экспериментах по изучению перехода аномального тлеющего разряда с полым катодом в дуговой разряд с распределенной термоэмиссией катода был получен рост тока от 1 до 100 А за время $\sim 150 \mu \mathrm{s}[2]$.

Полученные результаты подтвердили возможность реализации импульсно-периодического режима сильноточного разряда с полым самонакаливаемым катодом с частотой до нескольких килогерц. Интерес к такому режиму разряда обусловлен повышенным напряжением горения импульсного разряда, что приводит к росту температуры электронов и плотности анодной плазмы. Концентрация атомарного азота в плазме, генерируемой импульсным сильноточным электронным пучком в азотно-аргоновой смеси, существенно выше, чем в плазме непрерывного пучка [3]. Импульсный разряд с током $\sim 100 \mathrm{~A}$ стабильно горит в диапазоне длительностей 0.1-1 ms, тогда как в непрерывном разряде с ростом тока значительно возрастает уровень модуляции тока и напряжения в результате развития двухпотоковой неустой- чивости [4]. Эти свойства импульсно-периодического режима разряда делают перспективным его использование в системах реактивного ионно-плазменного нанесения покрытий.

Динамика температурного режима катода при совместном горении слаботочного разряда постоянного тока, который обеспечивает начальную температуру катода, и сильноточного импульсно-периодического разряда (ИПР) требует дополнительного изучения, поскольку она оказывает существенное влияние на напряжение горения импульсного разряда. В настоящей работе исследовано влияние величины тока вспомогательного разряда, частоты повторения импульсов, длительности и амплитуды импульсного тока на напряжение горения сильноточного разряда и проведены оценки теплового режима катода, объясняющие полученные результаты.

\section{1. Методика эксперимента}

Схема экспериментальной системы показана на рис. 1. В качестве полого катода 1 использовалась молибденовая трубка диаметром $10 \mathrm{~mm}$ с толщиной стенки $0.6 \mathrm{~mm}$, которая размещалась в водоохлаждаемом корпусе 2 соосно с тепловым экраном 3 диаметром $20 \mathrm{~mm}$. Анод 4 из нержавеющей стали в форме диска диаметром $200 \mathrm{~mm}$ и толщиной $\sim 15 \mathrm{~mm}$ размещался на расстоянии $\sim 300 \mathrm{~mm}$ от катода. Промежуточный электрод 5 находился под плавающим потенциалом. На внешней поверхности корпуса располагались 1-2 кольцевых постоянных магнита 6 из SmCo-сплава, обеспечивавших максимальную индукцию на оси катода 50-80 mT. Локальное неоднородное магнитное поле позволяло фиксировать положение активной зоны разряда независимо от величин тока и потока газа и стабилизировать разряд. Поток аргона в катодную полость регулировался в диапазоне $25-100 \mathrm{~cm}^{3} / \mathrm{min}$, давление газа в вакуумной камере составляло при этом около 0.04-0.3 Pa. 


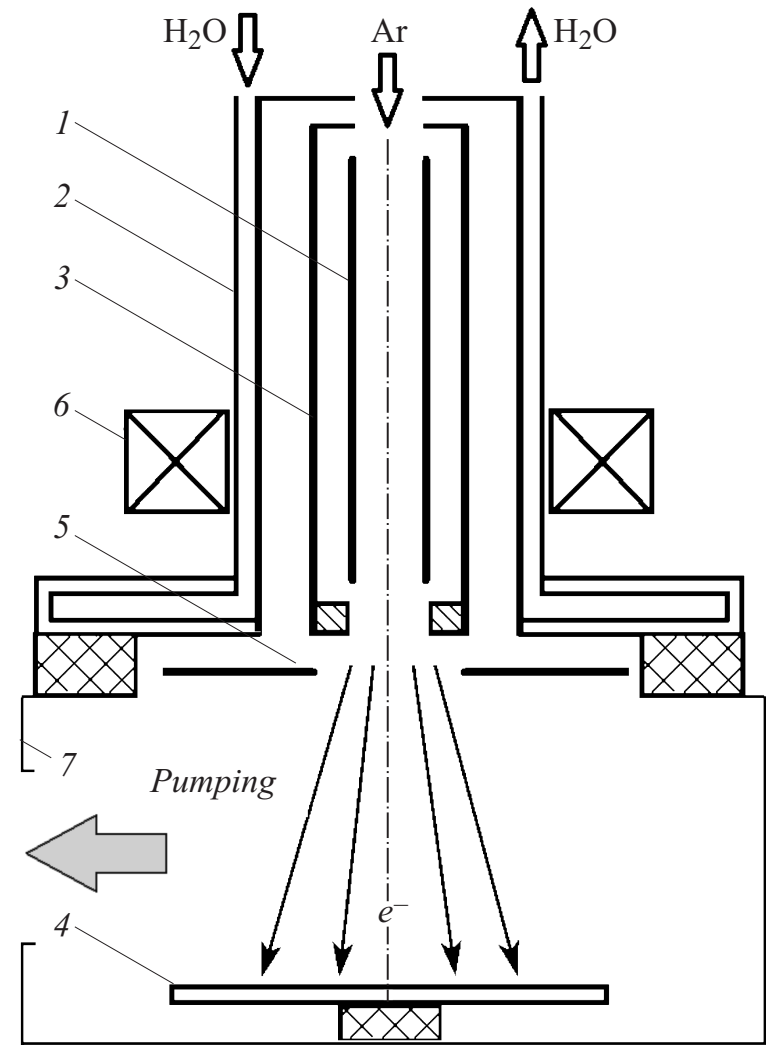

Рис. 1. Схема разрядной системы. 1 - катод, 2 - корпус, 3 экран тепловой, 4 - анод, 5 - промежуточный электрод, $6-$ магнит, 7 - камера вакуумная.

Постоянный ток $I_{d}$ разряда (РПТ) регулировали в пределах 1-5 А с помощью стабилизатора тока. Импульсный разряд с током $I_{m}=10-100 \mathrm{~A}$ возбуждали с регулируемой частотой $f(10-1000 \mathrm{~Hz})$ и длительностью импульсов $\tau(0.1-1 \mathrm{~ms})$ подачей на разрядный промежуток импульсов напряжения амплитудой $U_{m}=200-500 \mathrm{~V}$. Возможное сочетание параметров ИПР ограничивалось максимальной величиной среднего тока 4 А. Для регистрации и измерения импульсов тока и напряжения использовали осциллограф Тектроникс TPS2084.

Максимальная температура катода измерялась в разрядной системе без магнитного поля, размещенной непосредственно в вакуумной камере, через прорезь $3 \times 60 \mathrm{~mm}$ в тепловом экране. Для измерений использовался пирометр частичного излучения „Луч“ (ИТФ „Рида-С“) с эффективной длиной волны $0.92 \mu \mathrm{m}$, работавший в режиме измерения максимальной температуры. Расстояние между пирометром и катодом составляло $250 \mathrm{~mm}$. Измерения проводились через прозрачное для данной длины волны кварцевое смотровое окно.

\section{2. Результаты эксперимента}

Характерные осциллограммы тока и напряжения ИПР, полученные при одновременном горении в про- межутке РПТ с током $I_{d}=2 \mathrm{~A}$, приведены на рис. 2 . Длительность фронта импульса напряжения не превышала $10 \mu \mathrm{s}$, напряжение горения незначительно возрастало на стадии установления разряда, а затем стабилизировалось. На рис. 3 приведены вольтамперная характеристика (BAX) самостоятельного РПТ (кривая 1) и зависимость напряжения на промежутке в паузе между импульсами $U_{x}$ при совместном горении РПТ и ИПР от величины среднего тока $I_{a}=I_{d}+I_{m} f \tau$ (кривая 2), для которых характерно монотонное уменьшение напряжения с током. Особенностью зависимости 2 является уменьшение напряжения до значений, соответствующих сильноточному режиму постоянного тока $(30 \mathrm{~V})$, при относительно малом токе $\left(I_{a}=6 \mathrm{~A}\right)$. Средняя мощность, рассеиваемая при горении разрядов, линейно зависит от величины тока (рис. 3). На рис. 4 показаны ВАХ импульсного разряда $(\tau=220 \mu \mathrm{s})$, характер изменения которых зависит от частоты повторения импульсов. При меньших токах зависимости являются растущими,

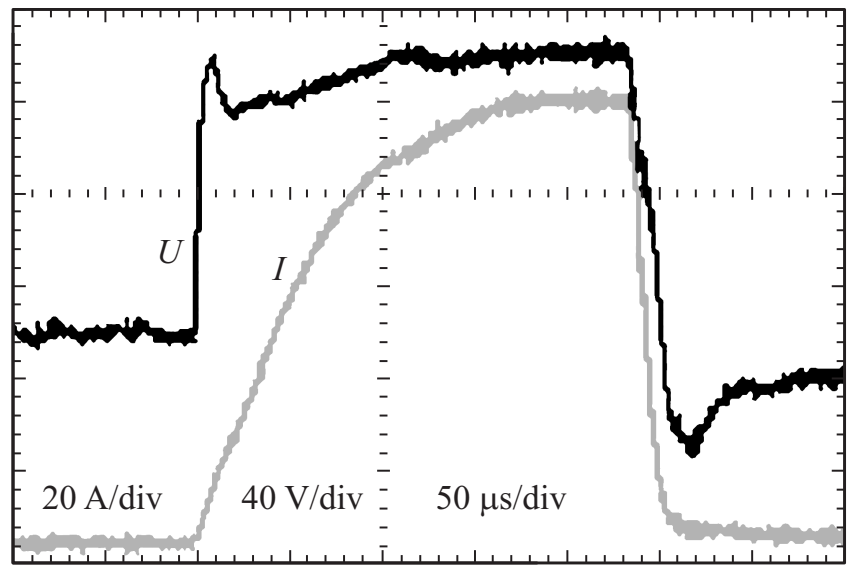

Рис. 2. Осциллограммы тока и напряжения. $I_{d}=2 \mathrm{~A}$, $Q=1 \mathrm{~cm}^{3} / \mathrm{s}$, Ar.

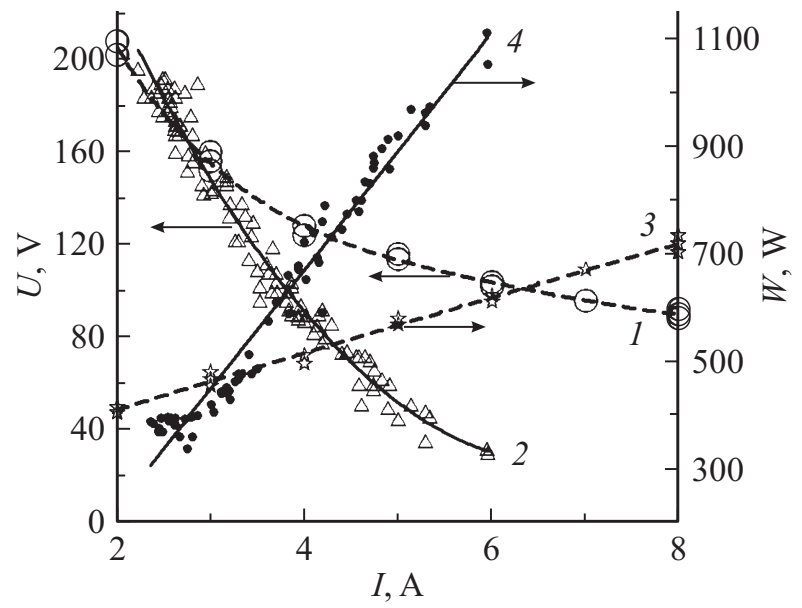

Рис. 3. Зависимости: $1-\mathrm{BAX}$ РПТ; $2-$ напряжения $U_{x}$ от среднего тока $I_{a}$; средней мощности разряда: 3 - от тока РПТ $I_{d}$ и 4 - от среднего тока $I_{a}$ в цепи катода. $f=10-200 \mathrm{~Hz}$, $\tau=220 \mu \mathrm{s} . I_{d}=2 \mathrm{~A}$. 


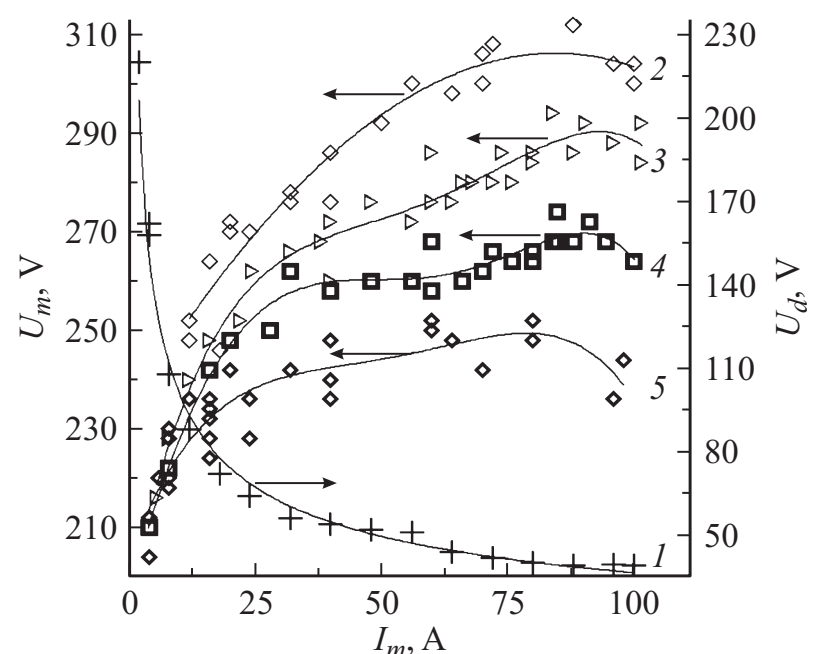

Рис. 4. ВАХ: $1-$ РПТ, $2-5-$ ИПР. $f=2-10,3-100$, $4-150,5-200$ Hz. $\tau=220 \mu \mathrm{s}, I_{d}=2 \mathrm{~A}$.

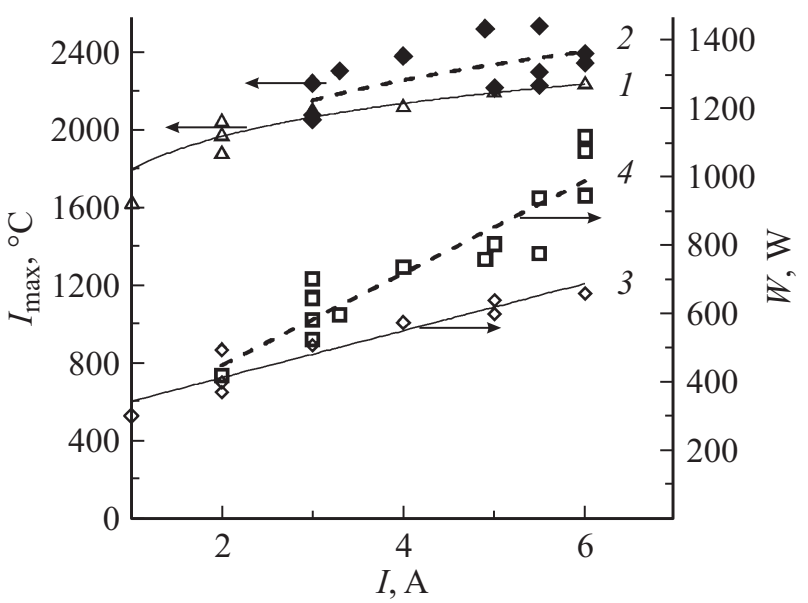

Рис. 5. Зависимости: 1,2 - максимальной температуры катода и 3,4- мощности от среднего тока $I_{a}: 1,3-$ РПТ и $2,4-$ ИПР $\left(I_{d}=2 \mathrm{~A}, \tau=200-500 \mu \mathrm{s}, f=200-1000 \mathrm{~Hz}\right)$. $B=0 \mathrm{mT}$.

a в области больших токов наблюдается насыщение зависимостей и последующий спад напряжения. С увеличением частоты напряжение горения ИПР уменьшается. Напряжение горения сильноточного самостоятельного РПТ монотонно убывает с ростом тока (кривая 1).

Зависимость температуры внешней поверхности катода и мощности, рассеиваемой в разряде, от тока РПТ и среднего тока катода при одновременном горении РПТ и ИПР представлена на рис. 5. Разброс значений температуры и мощности для ИПР обусловлен тем, что измерения при заданном среднем токе $I_{a}$ проводились для различных сочетаний параметров импульсного режима, отличающихся величиной напряжения горения разряда (рис. 4). Отметим, что приводимые расчетные значения мощности соответствуют полной мощности, потребляемой в разряде, при этом 60-80\% мощности в разряде такого типа может выделяться на аноде [5].

\section{3. Обсуждение результатов}

BAX непрерывного разряда с полым катодом в используемом диапазоне токов 1-100А обычно имеют U-образный вид. Снижение напряжения горения с ростом тока обусловлено переходом катода из режима ионно-электронной эмиссии в более эффективный термоэмиссионный режим. Рост напряжения на правой ветви ВАХ связан с нехваткой газа в полости и переходом разряда в режим горения в парах материала катода. При использовании материала катода с высоким давлением насыщенных паров, каким является молибден, растущая правая ветвь не наблюдалась. Как показал эксперимент, при совместном горении слаботочного и сильноточного ИПР вид ВАХ импульсного разряда зависит от параметров импульсно-периодического режима, влияющих на интегральную температуру катода. Рис. 4 иллюстрирует наличие как растущих, так и падающих участков ВАХ ИПР, причем немонотонная зависимость характеризуется наличием максимума, а не классического минимума.

Оценки, проведенные с использованием соотношения Ричардсона-Дэшмана, показывают, что для увеличения тока эмиссии молибденового катода на два порядка величины необходимо повысить температуру рабочей поверхности катода на $600 \mathrm{~K}$. В условиях эксперимента это достигается при постоянном среднем токе разряда 1 А и импульсном токе $100 \mathrm{~A}$. С учетом того, что размер активной зоны на поверхности катода с ростом тока разряда сокращается [6], прирост температуры катода $\Delta T$ на стадии роста тока может быть меньшим. Полученная величина $\Delta T$ находится в удовлетворительном соответствии с результатами расчета теплового режима самонакаливаемого катода [1]. Изменение температуры поверхности катода в процессе остывания при условии, что за время импульса прогревается слой, толщина которого много меньше толщины стенки катода, можно оценить с использованием соотношения [7]

$$
T(0, t)=2(q / \lambda)(a / \pi)^{1 / 2}\left[t^{1 / 2}-(t-\tau)^{1 / 2}\right],
$$

где $q$ - плотность мощности на катоде, $a-$ коэффициент температуропроводности, $a=\lambda /\left(\rho c_{p}\right), \rho-$ плотность, $c_{p}$ - удельная теплоемкость, $\lambda-$ коэффициент теплопроводности материала катода, $t$ - время с момента подачи импульса, $\tau$ - длительность импульса.

Оценка времени, за которое поверхность катода остывает до температуры, соответствующей начальной температуре катода на фронте импульса напряжения, дает значение $t_{1} \sim \tau$. За счет быстрого отвода тепла в объем катода поверхность катода остывает за время, много меньшее длительности паузы, которая в экспериментах составляла от 4 до $40 \mathrm{~ms}$.

Установившаяся интегральная температура катода определяется суммарным энерговкладом РПТ и ИПР разрядов. Более низкие значения напряжения $U_{x}$ на промежутке в паузе между импульсами от суммарного тока (рис. 3,2) по сравнению с ВАХ РПТ (рис. 3,1) 
обусловлены тем, что разряд в паузе стабилизирован по току, величина которого меньше, чем средний ток, определяющий температуру катода, т. е. разряд постоянного тока в паузе является несамостоятельным.

Растущий характер ВАХ ИПР (рис. 4) в области меньших токов обусловлен необходимостью импульсного нагрева катода до более высоких температур для увеличения тока, что обеспечивается ростом напряжения; причем с ростом амплитудного тока скорость роста напряжения снижается, а при большой частоте повторения импульсов меняет знак. Зависимость хода BAX от амплитудного тока и частоты повторения импульсов обусловлена изменением среднего тока разряда и соответственно изменением начальной температуры катода. С ростом среднего тока увеличивается начальная температура катода, поэтому скачок температуры, обеспечивающий требуемый амплитудный ток, требует меньшего роста напряжения, а при наибольших средних токах прирост начальной температуры катода оказывается достаточным, чтобы обеспечить рост импульсного тока при меньшем напряжения горения. Характер зависимостей становится падающим, как в РПТ.

Стабилизация напряжения и тока за время импульса является свидетельством того, что разряд переходит в квазистационарное состояние с напряжением горения, многократно превышающим напряжение РПТ с таким же током. Это связано с тем, что в отличие от РПТ с установившейся интегральной температурой катода баланс энергий на поверхности катода включает поток энергии в объем катода. Пренебрегая потерями энергии на конвективную теплопередачу и потерями на излучение, преимущественно поглощаемое стенками полости, для напряжения горения разряда $U$ по аналогии с [8] можно записать следующее соотношение:

$$
e U=\phi+3 / 2 k T_{e}+\varepsilon_{p}+\varepsilon_{t},
$$

где $\phi$ - работа выхода электрона, $T_{e}$ - температура электронов в плазме, $k$ - постоянная Больцмана, $\varepsilon_{p}$ удельные потери энергии в плазме, $\varepsilon_{t}$ - удельные потери, обусловленные отводом тепла в объем.

Таким образом, процесс горения импульсного разряда можно разделить на две стадии: стадия нагрева поверхности катода и роста тока разряда и стадия, на которой температура поверхности катода и ток стабилизируются на уровне, зависящем от величины приложенного напряжения и обеспечивающем выполнение приведенного выше энергетического баланса. Следовательно, проведенные в [1] с использованием граничного условия второго рода оценки температурного режима катода применимы только на стадии установления разряда и должны быть скорректированы с учетом непостоянства плотности потока мощности на катоде, а для импульсов большей длительности на стадии установившегося разряда необходимо использовать граничные условия первого рода с учетом постоянной температуры поверхности катода. Отметим, что проведение уточненных тепловых расчетов выходит за рамки настоящей работы.
Измерения температуры катода показали, что с учетом погрешности, обусловленной перепадом температуры по толщине стенки и неравномерностью распределения эмиссии по длине катода, ее величина, как и следовало ожидать, соответствует среднему току разряда. Более высокая мощность, потребляемая в ИПР по сравнению с РПТ, приводит к более высокой интегральной температуре катода.

Представляет интерес оценка эффективности полого самонакаливаемого катода в импульсно-периодическом режиме. Как известно, эффективность катода оценивается отношением тока эмиссии электронов к мощности накала и для проволочных металлических термокатодов прямого накала эта величина составляет $2-10 \mathrm{~mA} /\left(\mathrm{cm}^{2} \cdot \mathrm{W}\right)$ [9]. Для вспомогательного разряда постоянного тока при изменении тока от $1 \mathrm{~A}\left(U_{d}=\right.$ $=200 \mathrm{~V})$ до $5 \mathrm{~A}\left(U_{d}=100 \mathrm{~V}\right)$ эффективность меняется в пределах $\sim 5-10 \mathrm{~mA} / \mathrm{W}$. Эффективность импульсного разряда с током $100 \mathrm{~A}$ при напряжении $200 \mathrm{~V}$, горящим на фоне разряда постоянного тока с мощностью $200 \mathrm{~W}$, также составляет около $5 \mathrm{~mA} / \mathrm{W}$. Таким образом, поскольку мощность вспомогательного разряда на два порядка ниже мощности импульсного разряда, эффективность импульсной эмиссии самонакаливаемого катода оказывается сопоставимой с эффективностью металлических катодов прямого накала в разрядах постоянного тока. В непрерывном режиме горения разряда с напряжением 50-100 V эффективность самонакаливаемого катода достигает $10-20 \mathrm{~mA} / \mathrm{W}$.

Следует отметить, что хотя высокое напряжение горения разряда в импульсно-периодическом режиме повышает плотность плазмы в анодной части разряда вследствие роста температуры электронов плазмы, однако при этом существенно сокращается время жизни катода. Если уменьшение массы TiN-катода, вызванное эрозией его внутренней поверхности, при горении РПТ с током 25 А происходит со скоростью $\sim 2.3 \cdot 10^{-7} \mathrm{~g} / \mathrm{C}$, то в ИПР с параметрами $I_{m}=400 \mathrm{~A}, f=100 \mathrm{~Hz}, \tau=100 \mu \mathrm{s}$ скорость эрозии катода составила $\sim 2.5 \cdot 10^{-6} \mathrm{~g} / \mathrm{C}[1]$.

\section{Заключение}

Возможность реализации сильноточного импульсного режима горения разряда с самонакаливаемым полым катодом обусловлена локализацией ионизационных и эмиссионных процессов в малом объеме катодной полости при повышенном давлении газа, что обеспечивает высокую частоту ионизации газа, быстрый уход ионов на катод и нагрев поверхностного слоя катода до высокой температуры.

Переход разряда с самонакаливаемым полым катодом из слаботочного режима с током $\sim 1$ А в сильноточный режим с током до $100 \mathrm{~A} \mathrm{в} \mathrm{трубках} \mathrm{диаметром} 10 \mathrm{~mm}$ при величине потока аргона $1 \mathrm{~cm}^{3} / \mathrm{s}$ происходит за время $\sim 100 \mu \mathrm{s}$, что определяет минимально возможную 
длительность и максимальную частоту повторения импульсов тока. Отвод тепла в объем после прекращения импульса происходит за время, сопоставимое с длительностью фронта импульса тока.

При длительностях импульса $\tau>0.1 \mathrm{~ms}$ разряд переходит в квазистационарный режим, при котором величина напряжения горения в несколько раз превышает напряжение горения постоянного разряда с тем же током. Характер вольт-амперной характеристики импульсного разряда зависит от средней величины тока, протекающего в разрядном промежутке, который определяет начальную температуру катода на фронте импульса. С ростом величины импульсного тока и частоты повторения импульсов первоначально растущая зависимость напряжения импульсного разряда от тока становится падающей.

Работа выполнена в рамках государственного задания ФАНО России (№ 115031110091).

\section{Список литературы}

[1] Гаврилов Н.В., Меньшаков А.И. // ЖТФ. 2016. Т. 86. Вып. 5. C. $30-36$.

[2] Гаврилов Н.В., Меньшаков А.И., Мамаев А.С., Липчак А.И. // Изв. вузов. Физика. 2014. Т. 57. № 11/3. С. 209-213.

[3] Гаврилов Н.В., Меньшаков А.И., Соломонов В.И., Липчак А.И. // Изв. вузов. Физика. 2015. Т. 58. № 9/2. С. 88-92.

[4] Goebel D.M., Jameson K.K., Katz I., Mikellides I.G. // Phys. Plasmas. 2007. Vol. 14. 103508. P. 1-15.

[5] Nerovnyi V.M., Khakhalev A.D. // J. Phys. D: Appl. Phys. 2008. V. 41. N 3. 035201 P. 2452-2459.

[6] Krishnan M., Jahn R.G., von Jaskowsky W.F., Clark K.E. // AIAA J. 1977. Vol. 15. N 9. P. 1217-1223.

[7] Hartmann W., Dominic V., Kirkman G.F., Gundersen M.A. // J. Appl. Phys. 1989. V. 65. P. 4388-4395.

[8] Kennedy R.V. // J. Phys. D: Appl. Phys. 2001. V. 34. P. 787-793.

[9] Кноль М., Эйхмейер И. Техническая электроника. М.: Энергия, 1971. Т. 1. 472 с. 